\title{
Immunogenicity Specimen Assessments Reason Not Done
}

National Cancer Institute

\section{Source}

National Cancer Institute. Immunogenicity Specimen Assessments Reason Not Done. NCI Thesaurus. Code C117565.

The rationale why an immunogenicity specimen assessment was not performed. 